\title{
Least Squares Magnetic-Field Optimization for Portable Nuclear Magnetic Resonance Magnet Design
}

\author{
Jeffrey L. Paulsen ${ }^{1,2}$, John Franck ${ }^{3}$, Vasiliki Demas ${ }^{4}$, and Louis-S. Bouchard ${ }^{\text {5***}}$ \\ ${ }^{1}$ College of Chemistry, University of California, Berkeley, CA 94720 USA \\ ${ }^{2}$ Materials Sciences Division, Lawrence Berkeley National Laboratory, Berkeley, CA 94720 USA \\ ${ }^{3}$ Schlumberger-Doll Research, Cambridge, MA 02139 \\ ${ }^{4} \mathrm{~T} 2$ Biosystems, Cambridge, MA 02141 USA \\ ${ }^{5}$ Department of Chemistry and Biochemistry, University of California, Los Angeles, CA 90095 USA
}

\begin{abstract}
Single-sided and mobile nuclear magnetic resonance (NMR) sensors have the advantages of portability, low cost, and low power consumption compared to conventional high-field NMR and magnetic resonance imaging (MRI) systems. We present fast, flexible, and easy-to-implement target field algorithms for mobile NMR and MRI magnet design. The optimization finds a global optimum in a cost function that minimizes the error in the target magnetic field in the sense of least squares. When the technique is tested on a ring array of permanent-magnet elements, the solution matches the classical dipole Halbach solution. For a single-sided handheld NMR sensor, the algorithm yields a $640 \mathrm{G}$ field homogeneous to $16100 \mathrm{ppm}$ across a $1.9 \mathrm{~cm}^{3}$ volume located $1.5 \mathrm{~cm}$ above the top of the magnets and homogeneous to $32200 \mathrm{ppm}$ over a $7.6 \mathrm{~cm}^{3}$ volume. This regime is adequate for MRI applications. We demonstrate that the homogeneous region can be continuously moved away from the sensor by rotating magnet rod elements, opening the way for NMR sensors with adjustable "sensitive volumes."
\end{abstract}

\section{Key Words}

Least-squares optimization, magnetic devices, magnetic resonance, magnetostatics, nuclear magnetic resonance.

*** Corresponding author: L.-S. Bouchard (e-mail:bouchard@chem.ucla.edu). 


\section{Introduction}

DURING the last decade, a wide range of transportable, portable, and single-sided nuclear magnetic resonance (NMR) sensors and methodologies have been developed [1]-[12]. The interest in such devices, as potential standalone or complementary alternatives to conventional magnetic resonance imaging (MRI), arises from the obvious advantages of lower costs, portability, access to immovable arbitrary-sized objects, and scanning in the field. Mobile NMR measurements of, for example, relaxation times or MRI are currently conducted for applications within the materials sciences [13]-[20]. Recently, advances in methodology and hardware construction have allowed for high-resolution NMR spectroscopy in one-sided, portable NMR systems [21], [22].

Portable systems feature strong magnets that produce either a remote homogeneous field or some type of natural gradient. The field homogeneity and size of the homogeneous region come at the expense of the size of these systems or the overall field strength. For example, the NMR-MOLE is a $6 \mathrm{~kg}, 20 \mathrm{~cm}$ diameter magnet array that produces a $6.2 \mathrm{~cm}$ sensitive volume of $15000 \mathrm{ppm}$ homogeneity and a field strength of $767 \mathrm{G}$ located $1 \mathrm{~cm}$ away from the surface of the instrument [11]. By comparison, the original NMR-MOUSE weighs $2.5 \mathrm{~kg}$, has a $3.1 \mathrm{~cm}$ height and a $5.5 \mathrm{~cm}$ diameter, and produces a field strength of $5000 \mathrm{G}$ at the surface, but the sensitive volume is a thin, $1 \mathrm{~mm} \times 7 \mathrm{~mm} \times 3.5 \mathrm{~mm}$ slice in the center of the magnet [2]. Complementary to and independent of the magnet designs, several ex situ NMR methodologies were developed recently [23], [24] with the aim of relaxing hardware design and construction requirements in order to produce 
larger effective sensitive volumes. Such methodologies are based on the application of time modulated RF and static field gradient pulses that generate spatially dependent phase corrections, without altering the chemical shift signature of the sample under study. For instance, ex situ hardware matching [21], [23], [25] corrects the spin phases using crafted radio frequency (RF) fields whose spatial gradient matches that of the static field gradient. Shim pulses [24] achieve similar phase corrections by modulating the imaging gradients during the application of $\mathrm{RF}$ pulses without needing a particular spatial variation of the RF field. While these techniques help in correcting spin dephasing to some extent, and may improve the apparent homogeneity of a magnet, a relatively homogeneous magnet design is still a required starting point for individual applications.

We introduce a permanent-magnet design method which is simple in concept and implementation, in the sense that it can easily be programmed on a computer and optimizations are produced in a few minutes. The user specifies a target field over a volume, and the algorithm outputs a globally optimal arrangement of dipole sources based on desired tradeoffs between design efficiency and accuracy. This approach ultimately yields optimal magnet rod sizes and orientations. Such magnet rods are nowadays available at low cost from many commercial vendors in several different sizes. Moreover, their assembly as elements of an array is far less dangerous than the gluing of permanent-magnet (PM) blocks.

\section{Theory}


In this section, the general least squares problem that minimizes the norm squared of the difference between the trial and target fields is described. A more compact, but less intuitive, version is given in the Appendix.

To generate a field $\mathbf{B}(\mathbf{r})$ that best matches a target field, $\mathbf{t}(\mathbf{r})$, in the sense of least squares, one minimizes the volume integral of the norm squared of the difference between the fields over the region of interest (ROI)

$$
C=\int_{V_{R O I}}\|\vec{B}(\mathbf{r})-\vec{t}(\mathbf{r})\|^{2} d \mathbf{r} .
$$

We describe the trial field, as a linear combination of basis fields

$$
\vec{B}(\mathbf{r})=\sum_{m} g_{m} \vec{b}_{m}(\mathbf{r})
$$

For our particular optimizations, we use bases of two-dimensional infinitesimal dipoles. A linear combination of an $\mathbf{x}$ and a $\mathbf{y}$ oriented dipole centered at the appropriate position fully describes each magnet's strength and orientation, and an infinitely long cylinder uniformly magnetized perpendicular to its axis will reproduce this field. The cylinder's field strength can be scaled to match the dipole's without changing the shape of the field outside of it by scaling its radius as the square root of the dipole's strength. The field of such a cylinder magnetized along the $\mathrm{x}$-axis is given in cylindrical coordinates by (3), where is the remanent field of the PM block, is the cylinder's radius, and $S_{\text {and }} \theta_{\theta}$ are the radial and angular coordinates, respectively

$$
\mathrm{B}=\frac{B_{r} R^{2}}{2 s^{2}}(\cos (\theta) \hat{\mathrm{s}}+\sin (\theta) \hat{\theta}) .
$$


Physical implementations employing rod lengths significantly longer than the maximum distance of any rod center to the target field will reproduce these designs within a scaling factor of the field strength at a small loss of homogeneity as shown in Section III-B.

By substituting (2) into (1), we get

$$
C=\mathrm{g}^{T} \mathrm{Mg}-2 \mathrm{~g}^{T} \mathrm{~m}+t
$$

where $t$ is a scalar, $\mathbf{M}$ is an $\mathrm{n} \mathrm{x} \mathrm{n}$ symmetric matrix and $\mathrm{m}$ an $n$ long vector, $n$ being the number of basis fields, and these are defined by the appropriate overlap integrals where $\mathrm{V}$ is the volume of the target region:

$$
\begin{aligned}
M_{i, j} & \equiv \int_{V} \vec{b}_{i}(\mathbf{r}) \cdot \vec{b}_{j}(\mathbf{r}) d \mathbf{r} \\
m_{i} & \equiv \int_{V} \vec{b}_{i}(\mathbf{r}) \cdot \vec{t}(\mathbf{r}) d \mathbf{r} \\
t & \equiv \int_{V} \vec{t}(\mathbf{r}) \cdot \vec{t}(\mathbf{r}) .
\end{aligned}
$$

To determine the extrema with respect to variations in the coefficients $\left\{\mathrm{g}_{\mathrm{i}}\right\}$, we differentiate (4) with respect to $g_{k}$ and use the fact that $\mathbf{M}$ is a symmetric matrix to get

$$
\frac{\partial C}{\partial g_{k}}=\sum_{i} 2 M_{k, i} g_{i}-2 m_{k} .
$$

Setting (6) equal to zero for all possible yields

$$
\mathrm{g}=\mathrm{M}^{-1} \mathrm{~m} \text {. }
$$


If $\mathbf{M}$ is not singular and hence the basis fields are linearly independent, the solution is optimal. This point, when it exists, must be a global minimum with respect $\left\{g_{i}\right\}$ to because the original cost function, (1), is bounded from below and is quadratic with respect to the optimized coefficients. If $\mathbf{M}$ is an ill-conditioned or even a singular matrix, or yields unrealizable or inefficient designs then matrix regularization or constrained optimization can overcome these issues.

\section{A. Optimizing for Dipole Orientation: Applying Multiple Quadratic Equality}

\section{Constraints}

Tasks such as determining optimal magnetization angles given fixed magnet block shapes, positions, and strengths; or reproducing a different field with a preexisting adjustable magnet using our rod implementation scheme, require calculating optimal magnetization orientations given fixed strengths. Here, (4) must

be optimized under the equality constraints, (8), where each $\alpha_{j}^{2}$ is a positive scalar fixing a rod's magnitude and the $n \times n$ matrices $\mathbf{K}_{\mathrm{j}}$ are symmetric and positive definite where $n$ is the number of basis fields

$$
\left\{\alpha_{j}^{2}=\mathrm{g}^{T} \mathbf{K}_{j} \mathrm{~g}\right\}
$$

In our application, has the diagonal elements corresponding to the $j$ th rod's $x$ and $y$ dipole fields set to 1 with the remaining elements set to 0 .

The case of a single quadratic constraint has been worked out for various applications, see for example [26]. The case of arbitrarily many quadratic constraints adds some complications, but this more general case is also tractable. 
Following Golub [26], we determine the constrained optimum via the method of Lagrange multipliers. The Lagrangian is

$$
\varphi=\mathrm{g}^{T} \mathbf{M g}-2 \mathrm{~g}^{T} \mathrm{~m}+\sum_{j} \lambda_{j}\left(\mathrm{~g}^{T} \mathbf{K}_{j} \mathrm{~g}-\alpha_{j}^{2}\right)
$$

where $\lambda=\left\{\lambda_{1}, \lambda_{2}, \ldots, \lambda_{n}\right\}$ are the Lagrange multipliers. The extremum of (9) is attained when its gradient with respect to $g$ is zero, and is given by

$$
\mathrm{g}_{o p t}=\left(\mathbf{M}+\sum_{j} \lambda_{j} \mathbf{K}_{j}\right)^{-1} \mathrm{~m}
$$

where we have used the fact that $\mathbf{M}$ and $\mathbf{K}_{\mathbf{j}}$ are symmetric, positive definite matrices. Since the form of the Lagrangian is the same as our original cost function, this is still (7) but with a modified matrix.

Solving for the Lagrange multipliers by substituting the solution for $g$ into the constraints (8) yields an expression that does not have a trivial solution. A NewtonRaphson algorithm canbe applied to solve for $\lambda$. We approximate optimal coefficients, (10), by substitution into the constraints, (8), to first order in $\lambda$. Specifically, the matrix equation $(\mathbf{I}-a \mathbf{A})^{-1}$ can be approximated by $\mathbf{I}+a \mathbf{A}$ for small values of $a$. Thus, to first order in $\lambda$, the optimal coefficients are

$$
\mathrm{g}_{\text {opt }}=\mathbf{M}^{-1} \mathbf{m}-\sum_{j} \lambda_{j} \mathbf{M}^{-1} \mathbf{K}_{j} \mathbf{M}^{-1} \mathbf{m}
$$

Substituting (11) into (8) and retaining only the zeroth and first order terms in $\lambda$, our constraints can be approximated by 


$$
\begin{aligned}
\alpha_{k}^{2}=\left(\mathbf{M}^{-1} \mathrm{~m}\right)^{T} & \mathbf{K}_{k}\left(\mathbf{M}^{-1} \mathbf{m}\right) \\
& -2 \sum_{j} \lambda_{j}\left(\mathbf{M}^{-1} \mathbf{m}\right)^{T} \mathbf{K}_{k} \mathbf{M}^{-1} \mathbf{K}_{j}\left(\mathbf{M}^{-1} \mathbf{m}\right) .
\end{aligned}
$$

The solution for $\lambda$ now reduces to solving a matrix equation

$$
\mathbf{L} \lambda-\mathrm{p}=-\mathrm{A}
$$

where the components are

$$
\begin{aligned}
A_{j} & =\alpha_{j}^{2} \\
p_{j} & =\left(\mathbf{M}^{-1} \mathbf{m}\right)^{T} \mathbf{K}_{j}\left(\mathbf{M}^{-1} \mathbf{m}\right) \\
L_{i, j} & =2\left(\mathbf{M}^{-1} \mathbf{m}\right)^{T} \mathbf{K}_{i} \mathbf{M}^{-1} \mathbf{K}_{j}\left(\mathbf{M}^{-1} \mathbf{m}\right) .
\end{aligned}
$$

The $\lambda$ obtained is not an exact solution, so the constraint is not immediately satisfied. To improve the compliance, the constraint methodology is applied iteratively to the cost function modified by the previously obtained Lagrangian, (14)

$$
{ }^{i+1} \mathbf{M}={ }^{i} \mathbf{M}+\sum_{j}{ }^{i} \lambda_{j} \mathbf{K}_{j}
$$

In our application, we found that this iterative technique consistently converged.

To match to a particular target field, we wanted to fit to the shape of the vector field rather than its particular magnitude, as in general one will not know the specific strength of the homogeneous region that a magnet can best produce. With equality constraints placed on the coefficients, their overall magnitude cannot adjust to best match the strength of the target field. This flexibility may be retained for quadratically constrained optimizations by placing a variable scalar term in the target field overlap vector $m$, substituting it with $s m$, and subsequently determining 
the optimal value for $s$. With this substitution, the iterative first-order equation for $\lambda$ becomes

$$
\mathbf{L} \lambda-\mathbf{p}=-\frac{1}{s^{2}} \mathrm{~A} .
$$

The general solution (16) follows if $\lambda$ is decomposed into the partial solutions for the Lagrange multipliers $\lambda_{p}$ and $\lambda_{A}$ as defined in (16)

$$
\begin{aligned}
\lambda & =\lambda_{p}-\frac{1}{s^{2}} \lambda_{A} \\
\mathrm{p} & =\mathbf{L} \lambda_{p} \\
\mathrm{~A} & =\mathbf{L} \lambda_{A} .
\end{aligned}
$$

The optimal match occurs when the original cost function is minimally perturbed so that the optimal solution obeys the constraints, and hence when $|\lambda|$ is minimized with appropriately defined constraint matrices $\mathbf{K}_{k}$. By explicitly writing out the norm of $\lambda$ as a scalar product of its solution and differentiating with respect to the field scalar, $s$, the optimum is attained for

$$
s=\sqrt{\frac{\lambda_{\alpha} \cdot \lambda_{\alpha}}{\lambda_{\alpha} \cdot \lambda_{p}}} .
$$

Equation (17) has no real solutions when $\lambda_{\alpha} \cdot \lambda_{p} \leq 0$. This condition would imply that an infinitely strong field would be optimal, a physically unrealistic situation given the application of finding optimal dipole orientations that matches a particular field. However, this condition can nonetheless arise in optimizations, and implementations of this technique should be careful to handle this condition. 


\section{Simulations}

We first verify the present technique in the context of designing ring magnet configurations, the solution of which is the well-known Halbach dipole [27]. We then apply the methodology to the design of a single-sided magnet array for use in mobile NMR. For this single-sided design, we demonstrate how to alter the field profile by only changing the rod orientations, and compare the effects of using finite versus infinite length rods for producing a variety of target fields using the quadratic equality constraint version of our optimization. All optimizations and field plots were computed with Matlab (The Mathworks, Natick, MA). The initial solutions for the proposed single-sided NMR array were checked using Comsol33's magnetostatics module (The COMSOL Group, Stockholm, Sweden) for consistency.

\section{A. Ring Magnets}

For ring-shaped arrays of magnets, an optimal solution for the magnetization is the Halbach dipole array [27]. This provides a check of our method against a known solution to this problem. The magnitude and orientation of a ring of $16 \mathrm{PM}$

elements, were optimized to reproduce a unit target field, $H_{x}=1$, over both a circular and a cross-shaped region of interest to test the effects of varied regions of interest. Fig. 1 illustrates the results from the direct application of (5) and (7), along with the analytical Halbach dipole configuration approximated with 16 magnet elements for comparison.

The optimization over the circular and cross-shaped regions show a remarkable similarity to the actual Halbach dipole configuration with respect to their fields and optimal magnetizations. These matches require an appropriate 
choice of the target field's region of interest and/or the use of matrix regularization, and yield designs with smaller homogeneous regions than the Halbach dipole that are within a part per ten thousand of the target field strength. There is also a $1 \%$ and $12 \%$ reduction in field strength relative to the Halbach dipole for the circular and cross-shaped target regions, respectively. Thus, this classic design, originally derived using analytical arguments [27], can alternatively be obtained using this relatively simple numerical method at a small cost in performance with respect to homogeneity and field strength.

The size of the circular target region had to be within a certain range (its radius had to be between about $60 \%$ to $90 \%$ that of the dipole positions) to produce designs resembling the Halbach dipole without matrix regularization. To obtain a Halbach-like design from the cross-shaped target region, the optimization matrix was regularized by adding the identity matrix to it in order to eliminate the very small negative eigenvalues from its diagonalization.

The difference in homogeneity between the Halbach and numerically optimized designs differ on the order of parts per ten thousand of the target field's strength and likely arise from numerical errors in calculating matrix terms and the matrix inverse. Deviations of this order of magnitude are not surprising given the optimization matrices involved are ill-conditioned, while the Halbach design comes from an exact analytical solution [27]. Practically, deviations on this level are not too important as variations in actual magnet blocks would cause larger variations in the field. Nonetheless, these variations indicate that the method requires improvements if one wanted to optimize for better homogeneities. Furthermore, 
analytically the matrices cannot have negative eigenvalues as the cost function is bounded from below, yet the numerically calculated matrix for the cross-shaped target region often does. This indicates that numerical errors can have significant qualitative effects, and leads to the need for matrix regularization.

\section{B. Design of a Single-Sided NMR Magnet}

We now demonstrate an application to the design of a portable, single-sided MRI sensor. A series of unconstrained optimizations identified an optimal magnet geometry and orientation of the magnet rods, which was then characterized by finite-element modeling. Subsequent optimizations demonstrate that sensitive volume can be moved by simply rotating the magnet rods.

For the initial optimization, the optimal dipole strengths and orientations were optimized for a series of possible placements of four dipoles via the least squares optimization method presented. The optimal dipole positions were chosen by selecting the best of these possible arrangements. Algorithms 1 and 2 describe the optimization scheme. Fig. 2 illustrates the possible dipole locations, the target field region, and the field zeroing region.

Dipole orientations and strengths were optimized for a main target field of unit strength and oriented parallel to the plane containing the magnets. ${ }^{1} \mathrm{An}$ additional optimization matrix and vector based on a zero target field over the field zeroing region were added at a small scaling factor determined by Algorithm $1\left(\mathrm{f}_{\mathrm{z}}=\right.$ $1.6 \times 10^{-4}$ ) to the main target field's optimization matrix and vector before

${ }^{1}$ The target field's orientation allows for the use of RF-coils with greater sensitivity. 
calculating the optimal coefficients to make the inverted matrix less ill-conditioned and improve the design's efficiency. To maximize design efficiency and maintain reasonable homogeneity, we used the largest possible scaling factor that kept the homogeneity to $\sim 1$ part per hundred, which is comparable in magnitude to the performance of previously published systems [11], [12], [28], [29].

From this series of arrangements, each with optimized dipole strengths and orientations, the best in terms of homogeneity, field strength, and stability was selected to obtain optimal dipole positions. The homogeneity is calculated as the RMS deviation of the field from the target field, which is Sqrt(C)/A where C is our cost function from (4) and is the area for the main target field. Equation (3) determines the field strength at the center of the target region for the rod radii and orientations corresponding to the optimal coefficients. The stability of a set of dipole positions is quantified by the magnitude of the gradient of the cost function with respect to changes in dipole position and magnetization $\left(\left|\nabla_{\mathbf{x}} C\right|\right.$ and $\left|\nabla_{\mathrm{g}} C\right|$, respectively), and is calculated by finite differences between the position's optimal design and its perturbations.

The best arrangement from our search is illustrated in Fig. 3, and these optimal dipole positions are also shaded in Fig. 2 to view it in relation to the original optimization parameters. The construction, performance, and application of this system will be presented in a future publication. Since the original optimization applies to infinitely long rods, we perform finite-element modeling of its finite rod implementation to validate the performance of this method (see Fig. 3). The dipoles 
are implemented with $18 \mathrm{~cm}$ long rods ${ }^{2}$ so that the length of any one rod is significantly longer than the distance of its center to the target field. In this case, the rod lengths are slightly more than 3 times longer than their distance to the target region. The optimal coefficients and available space lead to outer and inner rod radii of $2 \mathrm{~cm}$ and $1 \mathrm{~cm}$, and orientations off from coplanar by $24.1 \mathrm{deg}$ and $3.5 \mathrm{deg}$, respectively, as illustrated in Fig. 3. A Comsol33 simulation indicates that this configuration with rods whose remanent fields are $1.3 \mathrm{~T}$ yields a $640 \mathrm{G}, 1.9 \mathrm{~cm}$ sensitive volume with a $16100 \mathrm{ppm}$ homogeneity, or a $7.6 \mathrm{~cm}$ sensitive volume with a 32200 ppm homogeneity.

Finally, we fine tune the rod orientations of the previously optimized design to adjust for the effects of finite rod lengths, and demonstrate how this design can produce a movable sensitive volume by rotating the magnet rods with a series of leastsquares optimizations subject to multiple of quadratic equality constraints (see Section II-A). The target fields are identical in size (a $2 \mathrm{~cm} 1 \mathrm{~cm}$ slice in the plane bisecting the rod axes) and orientation to the original optimization, but vary in their distance from the array to produce a movable sensitive volume [see Fig. (4i)]. The optimizations accurately model and adjust for finite rod lengths, by using the basis fields of finite rods (numerically evaluated assuming $B_{r}=1.3 \mathrm{~T}$ ), and repeats these optimizations using an infinite rod basis for comparison. The equality constraints are necessary to use with a finite rod basis, as field shape, in addition to magnitude, varies with the rod's dimensions. Each rod's orientation is then modeled by the superposition of two basis functions: one for magnetization along $\mathbf{x}$ and one along $\mathbf{y}$.

2 While $18 \mathrm{~cm}$ long rods are hard to obtain and impractical to use, they can be realized by aligning two collinear $9 \mathrm{~cm}$ long rods. 
The variations are restricted to rod rotations only, by constraining the sum of squares of the pair of coefficients representing each rod. Since the magnitude of the coefficients are fixed and unable to adjust to the strength of the target field, the optimization adjusts the target field strength to minimize the perturbation to the original cost function. The technique's implementation is specified in Algorithm 3.

Fig. 4 details the constrained optimization's results for a range of target fields for both $18 \mathrm{~cm}$ and infinitely long rod bases. This optimization yields the appropriate orientations of finite rods to give homogeneous regions like those in Fig. (4i) at different distances from the array. However, very close target regions $(<2.9 \mathrm{~cm})$ exhibited dramatically worsened homogeneities, as shown in Fig. (4iii), where the algorithm fails to yield homogeneous solutions, and gives chaotic behavior, as seen in the plots of optimal field strengths, Fig. (4ii), and optimal angles, Fig. (4iv). Arrangements with the highest field strengths and best homogeneities occur around a target field distance of $3.5 \mathrm{~cm}$ (labeled b). This coincides with the original target region, for which the rod sizes and positions were optimized. Further out target regions correspond to regions where the fields of the individual rods weaken, so it is natural that the fields of the optimal solutions also weaken. Furthermore, the rods' relative sizes and positions cease to be optimal for more distant sensitive volumes, and the homogeneity tends to worsen. Fig. (4ii)(iv) also provides a way to compare the use of the infinite rod basis to its finite length implementation. Replacement with the $18 \mathrm{~cm}$ long rods effectively leads to the sensitive volume's strength to be scaled down, a small decrease in homogeneity, and very similar optimal rod orientations which differ more for distant sensitive 
volumes. Thus, when the algorithm is able to produce homogeneous regions and the target region is reasonably close $(<4.5 \mathrm{~cm})$, implementation of the infinite rod design with finite length rods yields the same design with a small cost to homogeneity and a rescaled sensitive volume strength. The differences become more pronounced at more distant sensitive volumes where the infinite rod approximation becomes worse. Thus, the field scaling factor $(\sim 0.5$ at $3 \mathrm{~cm}$ and $\sim 0.4$ at $10.5 \mathrm{~cm}$ ) decreases with distance, and the optimal angles become significantly different past $4.6 \mathrm{~cm}$ though only for the inner rods in this example.

\section{Discussion}

We presented a magnet design technique based on the least-squares fit of a target field and demonstrated its application to the design of ring magnets and a single-sided NMR magnet. The optimization finds a global optimum for the sizes and orientations of an array of cylindrical magnets, whose potential adjustability offers greater experimental flexibility and several advantages for implementation. The optimization itself is simple and compares well to similar methods, but requires appropriate application to yield useful results.

We demonstrated that this technique leads to good permanent magnet designs. Its reproduction of designs very similar to the Halbach dipole array, even with a cross-shaped target region, confirms that it leads to reasonable optimal solutions. The technique also produced a single-sided NMR magnet design with good performance in terms of the balance between field strength, homogeneity, and 
sensitive volume location, and demonstrated that this design can have the unique feature of a variable sensitive volume location.

The overall approach leads to designs based on cylindrical magnet rods, and, though this can lead to weaker field strengths due to not filling a maximal amount of volume with magnetic material, these designs have several significant advantages. Such magnet rods are commercially available over a range of sizes, but more importantly allow for significant adjustments by rotating the rods with an appropriately designed frame. An adjustable frame allows the rods to be initially oriented for an easier assembly and to be adjusted from their theoretically optimal orientations to compensate for variations in the magnets. With accurate field maps of the actual rods, these corrections could be directly calculated. In our specific application, the resulting adjustable sensitive volume gives greater experimental flexibility: closer sensitive volumes for greater signal or further out to probe deeper within the sample. Without this adjustability, previous magnets could only achieve this flexibility over sensitive slices instead of over volumes [2].

Our approach is unique in comparison to other magnet optimization techniques [8], [30]-[34] in that it combines a target field optimization that locates a global optimum with a few simple matrix calculations to the design of adjustable permanent magnet assemblies. Other linear programing based techniques like $\mathrm{l}_{\mathrm{p}}$ norm [30], [31] have been applied to coil designs and could provide an alternative optimization technique within our implementation strategy. Our approach most closely resembles the scalar potential approach [35] in that it finds an optimal linear combination of basis fields, but that technique instead yields pole-piece based 
designs and optimizes the lower order terms of an expansion of the target field instead of fitting it over a volume.

While our optimization technique is simple to implement, it requires the appropriate selection of magnet rods and their corresponding basis fields. The 2-D dipole basis is useful for the initial optimization because its field has a simple analytical form and the relative dipole magnitudes can be adjusted in their infinite length rod implementation by changing their relative radii. However, their finitelength rod implementations must have the rod lengths significantly longer than the distance of any rod center to the region of the target field. Subsequent optimizations with a finite rod basis can help adjust for errors introduced in the approximation, but these can only adjust their orientations since quadratic equality constraints need to be used with this basis. Any such quadratically constrained optimization requires multiple iterations to correctly determine the Lagrange multipliers and should optimize the target field strength so to minimize the applied Lagrange multipliers because the optimal field strength will vary depending on the basis and target field location. As done in Section III-B, a series of these constrained optimizations with finite and infinite length rod bases can show where the finite rod implementation causes more than just a rescaling of field strength and a slight loss in homogeneity by testing a range of target field distances or possible rod lengths. With a range of target field distances, we demonstrated that with our design significant errors occurred when some of the rod lengths became less than 2.7 times longer than the distance of their centers to the target region, ${ }^{3}$ while also showing

${ }^{3}$ We omit the effect of the rod radius becoming to large relative to length, 
how to produce a movable sensitive volume. A more direct comparison between the infinite and finite length rod fields, while useful, is difficult because the field within the plane bisecting the finite rod lacks a simple analytical solution and the magnitude of its field is radially asymmetric unlike for the infinite rod case. Therefore, it is simpler to test the practical effect on the array.

Matrix regularization techniques and efficiency-increasing measures are often needed to ensure that the optimal designs are reasonably robust and efficient, because the optimization matrix $\mathbf{M}$ is often ill-conditioned. In the case of optimizing the ring array of magnets to the cross-shaped target field, the original optimization matrix $\mathbf{M}$ is poorly conditioned, but with a matrix regularization consisting of adding the identity matrix to its $\mathbf{M}$ at a small scaling factor, the optimization still nearly reproduces a Halbach like array. In the second set of optimizations, the relative homogeneity and efficiency of the optimized single-sided NMR magnet was controlled by adding the optimization matrix for the field-zeroing region $\left(\mathbf{M}_{\mathbf{z}}\right)$ to the main target field's optimization matrix (M) at a weighting that gave the desired balance of field strength and homogeneity. Thus, instead of obtaining optimal designs with very homogeneous, but weak fields, we could control the trade-off between homogeneity and field strength.

Finally, our optimization strategy leaves the dipole positions in need of optimization. A global search over a fairly coarse grid yielded a good one-sided MR magnet design, while slightly denser grids did not yield significant improvements.

since physical considerations like avoiding rod overlap with each other or with the target region will keep the rod radii significantly smaller than the distance to the target field and hence rod length. 
To fine tune dipole positions more computationally efficient strategies could be used, potentially borrowing from the strategies employed in $\mathrm{l}_{\mathrm{p}}$-norm optimizations for magnet design [30].

\section{Appendix A: Optimization Applied Over Discrete Points}

The original cost function, (1), with the basis set expansion directly applied to it is

$$
C=\int_{V}\|\mathbf{\Gamma}(\mathbf{r}) \mathrm{g}-\mathrm{t}(\mathbf{r})\|^{2} d \mathbf{r} .
$$

where $\mathrm{g}$ is the vector of linear weightings, $\Gamma(\mathbf{r})$ is a matrix whose columns represent different basis functions and whose rows represent different components of the field, and $t(r)$ is the target field. If the minimization is over a discrete set of points $\{p\}$ and the field's components are Cartesian, the integral of the norm becomes a sum of the square of the field's vector components

$$
C=\sum_{\{p\}} \sum_{\{\alpha\}}[\boldsymbol{\Gamma}(\mathbf{p}) \mathrm{g}-\mathrm{t}(\mathbf{p})]_{\alpha}^{2} \text {. }
$$

The sum over points can be combined with the sum over field components to create a simplified matrix expression, (20), if we let the rows of $\Gamma$ and t span a more general space that sequentially lists the field components of every point

$$
C=\|\Gamma \mathrm{g}-\mathrm{t}\|^{2} .
$$


The optimal solution here is the classic least-squares fitting problem of linear algebra, for which there are many pre-existing routines. The matrix is the same as the one used in the $l_{p}$-norm method to relate constraints placed on the field to constraints on the optimal currents.

An experimental realization of the 4-elements cylindrical rod design proposed in this paper is to appear in [36].

\section{Acknowledgments}

This work was supported by the Director, Office of Science, Office of Basic Energy

Sciences, Materials Sciences and Engineering Division, of the U.S. Department of Energy under Contract DE-AC02-05CH11231. The authors would like to thank Prof. A. Pines for his support, stimulating discussions, and advice, and Prof. B. Blümich for stimulating discussions and advice.

\section{References}

[1] D. P. Murphy, "Advances inMWDand formation evaluation for 1995," World Oil, vol. 216, pp. 39-49, 1995.

[2] G. Eidmann, R. Savelsberg, P. Blümler, and B. Blümich, "The NMR MOUSE, a mobile universal surface explorer," J. Magn. Reson., A, vol. 122, pp. 104-109, 1996.

[3] P. J. McDonald, "Stray field magnetic resonance imaging," Progr. Nucl. Magn. Reson. Spectrosc., vol. 30, p. 69, 1997.

[4] E. Fukushima and J. Jackson, "Unilateral magnets: An idea and some history," NMR News Lett., vol. 490, pp. 40-42, 1999.

[5] G. Guthausen, A. Guthausen, F. Balibanu, R. Eymael, K. Hailu, U. Schmitz, and B. Blümich, "Soft-matter analysis by the NMR-MOUSE," Macromol. Mater. Eng., vol. 276-277, pp. 25-37, 2000.

[6] F. Bãlibanu, K. Hailu, R. Eymael, D. E. Demco, and B. Blümich, "Nuclear magnetic resonance in inhomogeneous magnetic fields," J. Magn. Reson., vol. 145, pp. 246-258, 2000. 
[7] F. Casanova and B. Blümich, "Two-dimensional Imaging with a singlesided NMR probe," J. Magn. Reson., vol. 163, pp. 38-45, 2003.

[8] A. E. Marble, I. V. Mastikhin, B. G. Colitts, and B. J. Balcom, "An analytical methodology for magnetic field control in unilateral NMR," J. Magn. Reson., vol. 174, pp. 78-87, 2005.

[9] J. Perlo, F. Casanova, and B. Blümich, "Single-sided sensor for high-resolution NMR spectroscopy," J. Magn. Reson., vol. 180, pp. 274-279, 2006.

[10] P. J. McDonald, P. S. Aptaker, J. Mitchell, and M. Mulheron, "A unilateral NMR magnet for sub-structure analysis in the built environment: The surface GARField," J. Magn. Reson., vol. 185, pp. 1-11, 2007. [11] B. Manz, A. Coy, R. Dykstra, C. D. Eccles, M. W. Hunter, B. J. Parkinson, and P. T. Callaghan, "A mobile one-sided NMR sensor with a homogeneous magnetic field: The NMR-MOLE," J. Magn. Reson., vol. 183, pp. 25-31, 2006.

[12] W. H. Chang, J. H. Chen, and L. P. Hwang, "Single-sided mobileNMR with a Halbach magnet," Magn. Reson. Imag., vol. 24, pp. 1095-1102, 2006.

[13] A. Guthausen, G. Zimmer, P. Blümler, and B. Blümich, "Analysis of polymer materials by surface NMR via the MOUSE," J. Magn. Reson., vol. 130, pp. 1-7, 1998.

[14] R. Haken and B. Blümich, "Anisotropy in tendon investigated in vivo by a portable NMR scanner, the NMR-MOUSE," J. Magn. Reson., vol. 144, pp. 195-199, 2000.

[15] H. Kühn, M. Klein, A. Wiesmath, D. E. Demco, B. Blümich, J. Kelm, and P. W. Gold, "The NMR-MOUSE: Quality control of elastomers," Magn. Reson. Imag., vol. 19, pp. 497-499, 2001.

[16] S. Anferova, V. Anferov, D. G. Rata, B. Blümich, J. Arnold, C. Clauser, P. Blümler, and H. Raich, "A mobile NMR device for measurements of porosity and pore size distributions of drilled core samples," Concepts Magn. Reson. B: Magn. Reson. Eng., vol. 23 B, pp. 26-32, 2004.

[17] R. L. Kleinberg, “Well logging," Encyclopedia of NMR, vol. 105, pp. 4960-4969, 1996.

[18] R. L. Kleinberg, "NMR well logging at Schlumberger," Concepts Magn. Reson., vol. 13, pp. 396-403, 2001.

[19] F. Casanova, J. Perlo, and B. Blümich, "Velocity distributions remotely measured with a single-sided NMR sensor," J. Magn. Reson., vol. 171, pp. 124-130, 2004.

[20] J. Perlo, F. Casanova, and B. Blümich, "Velocity imaging by ex-situ NMR," J. Magn. Reson., vol. 173, pp. 254-258, 2005. [21] J. Perlo, V. Demas, F. Casanova, C. A. Meriles, J. Reimer, A. Pines, and B. Blümich, "High-resolution NMR spectroscopy with a portable single-sided sensor," Science, vol. 308, p. 1279, 2005.

[22] J. Perlo, F. Casanova, and B. Blümich, "Ex situ NMR in highly homogeneous fields: spectroscopy," Science, vol. 315, pp. 1110-1112, 2007. 
[23] C. A. Meriles, D. Sakellariou, H. Heise, A. J. Moulé, and A. Pines, "Approach to high-resolution ex-situNMRspectroscopy," Science, vol. 293, pp. 82-85, 2001.

[24] D. Topgaard, R. W. Martin, D. Sakellariou, C. A. Meriles, and A. Pines, "'Shim pulses" for NMR spectroscopy and imaging," Proc. Nat. Acad. Sci. USA, vol. 101, pp. 17576-17581, 2004.

[25] L.-S. Bouchard and M. S. Anwar, "Synthesis of matched magnetic fields for controlled spin precession," Phys. Rev. B, vol. 76, p. 014430, 2007.

[26] G. H. Golub, "Some modified matrix eigenvalue problems," SIAM Rev., vol. 15, no. 2, pp. 318-334, 1973.

[27] K. Halbach, "Design of permanent multipole magnets with oriented rare earth cobalt materials," Nucl. Instrum. Methods, vol. 169, pp. 1-10, 1980.

[28] A. E. Marble, I. V. Mastikhin, B. G. Colpitts, and B. J. Balcom, "A compact permanent magnet array with a remote homogeneous field," J. Magn. Reson., vol. 186, no. 1, pp. 100-104, 2007.

[29] A. E. Marble, I. V. Mastikhin, B. G. Colpitts, and B. J. Balcom, "A constant gradient unilateral magnet for near-surface MRI profiling," J. Magn. Reson., vol. 183, pp. 228-234, 2006.

[30] H. Xu, S. M. Conolly, G. C. Scott, and A. Macovski, "Homogeneous magnet design using linear programming," IEEE Trans. Magn., vol. 36, no. 2, pp. 476-483, Mar. 2000.

[31] S. E. Ungersma, H. Xu, B. A. Chronik, G. C. Scott, A. Macovski, and S. M. Conolly, "Shim design using a linear programming algorithm," Magn. Reson. Med., vol. 52, pp. 619-627, 2004.

[32] R. Turner, "Gradient coil design: A review of methods," Magn. Reson. Imag., vol. 11, pp. 903-920, 1993.

[33] S. Russenschuck, "Synthesis, inverse problems and optimization in computational electromagnetics," Int. J. Numer. Model.: Electron.

Netw, Dev. Fields, vol. 9, pp. 45-57, 1996.

[34] B. Luong, J. C. Goswami, A. Seqginer, and D. Davies, "Optimal control technique for magnet design in inside-out nuclear magnetic resonance," IEEE Trans. Magn., vol. 37, no. 2, pp. 1015-1023, Mar. 2001. [35] A. E. Marble, I. V. Mastikhin, B. G. Colpitts, and B. J. Balcom, "Designing static fields for unilateral magnetic resonance by a scalar potential approach," IEEE Trans. Magn., vol. 43, no. 5, pp. 1903-1911, May 2007.

[36] J.-L. Paulsen et al., "Volume-selective magnetic resonance imaging using an adjustable, single-sided, portable sensor," PNAS, submitted for publication. 
Fig. 1. Optimal ring arrays of magnet rods that produce the uniform magnetic field, over their central regions. The fields are calculated assuming infinitely long rods, each with uniform remanent fields of $1.3 \mathrm{~T}$. The homogeneity of each field's magnitude within parts per hundred (pph), parts per thousand (ppt), and parts per ten-thousand (pptt) are indicated by the shaded regions. The circles indicate rod placement and sizes, and the arrows indicate the orientation of each rod's magnetization. We optimized using the presented technique over circular and cross-shaped regions as indicated by the unmasked areas (masked areas are indicated by crosshatching) and included the Halbach dipole solution for comparison. For both target regions, we obtain solutions with rod sizes and orientations similar to the Halbach dipole.

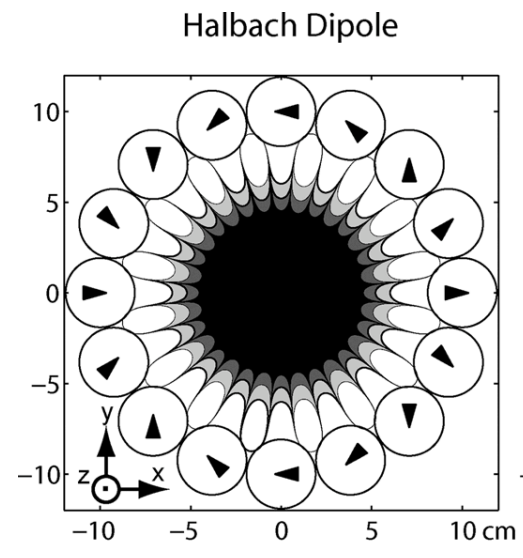

Field at Center: 3801.5 Gauss
Circular Target Region $\mathrm{R}=8 \mathrm{~cm}$

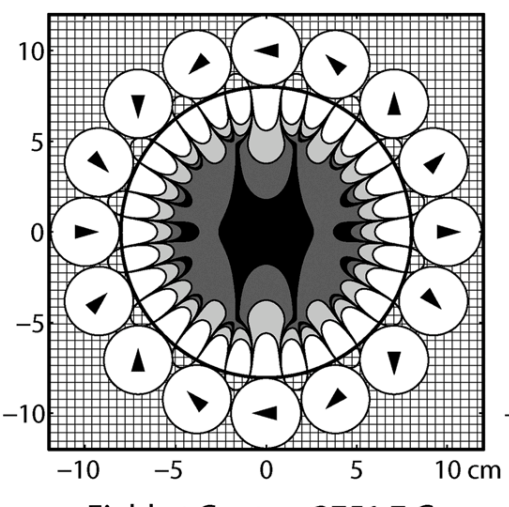

Field at Center: 3751.7 Gauss
Cross Target Region

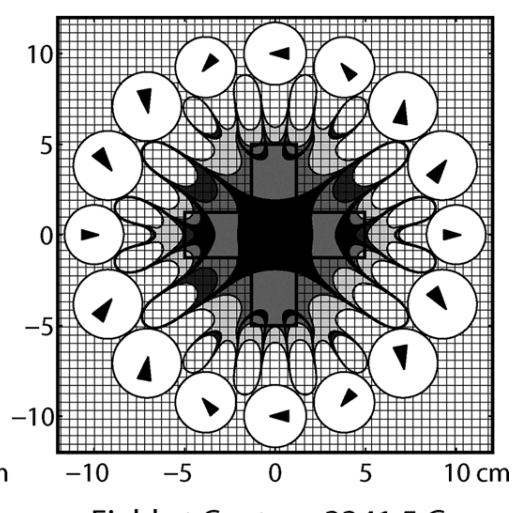

Field at Center : 3341.5 Gauss

$$
\operatorname{pptt}\left(10^{-4}\right) \square \operatorname{ppt}\left(10^{-3}\right) \square \operatorname{pph}\left(10^{-2}\right) \square
$$


Fig. 2. Optimization parameters for the initial design of a single-sided magnet for NMR. The target region for a homogeneous field is indicated by the white rectangle and the region for field minimization (field zeroing) by the hatched region. Circles represent possible dipole positions, where a possible combination of 4 dipoles obeying the appropriate symmetry for selection are shaded. The schematic illustrates the xy-plane of the system, where the rods producing the dipole fields extend to infinity along $+/ \_\mathbf{z}$.

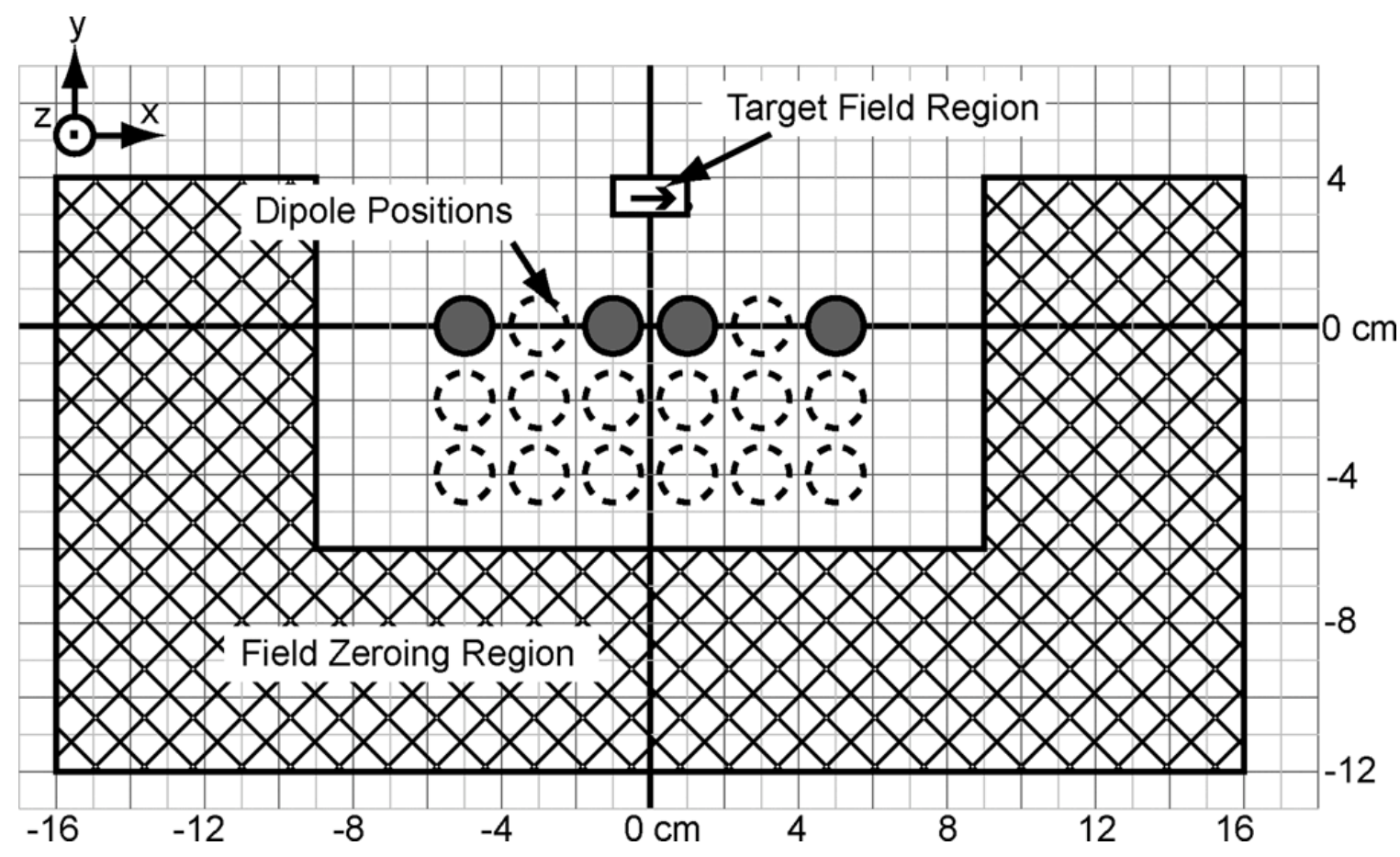


Fig. 3. The optimal magnet design implemented with $18 \mathrm{~cm}$ long magnet rods, each with a uniform remanent field of $1.3 \mathrm{~T}$, and modeled using Comsol33. The magnitude of the magnetic field is plotted over the slice bisecting the magnetic rods over a range of 550 to $710 \mathrm{G}$ with off-scale regions in white. The magnet rods are indicated by the gray cylinders and the orientation of their magnetization by the arrows.

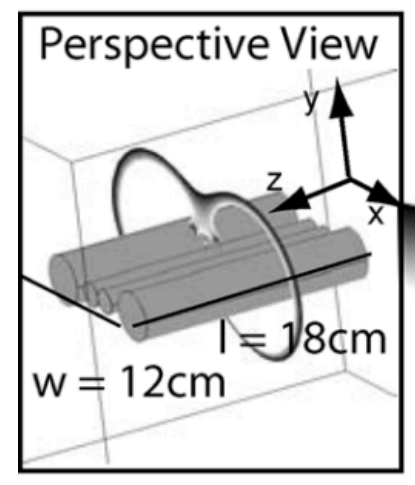

$|\mathrm{B}|$ (Gauss)

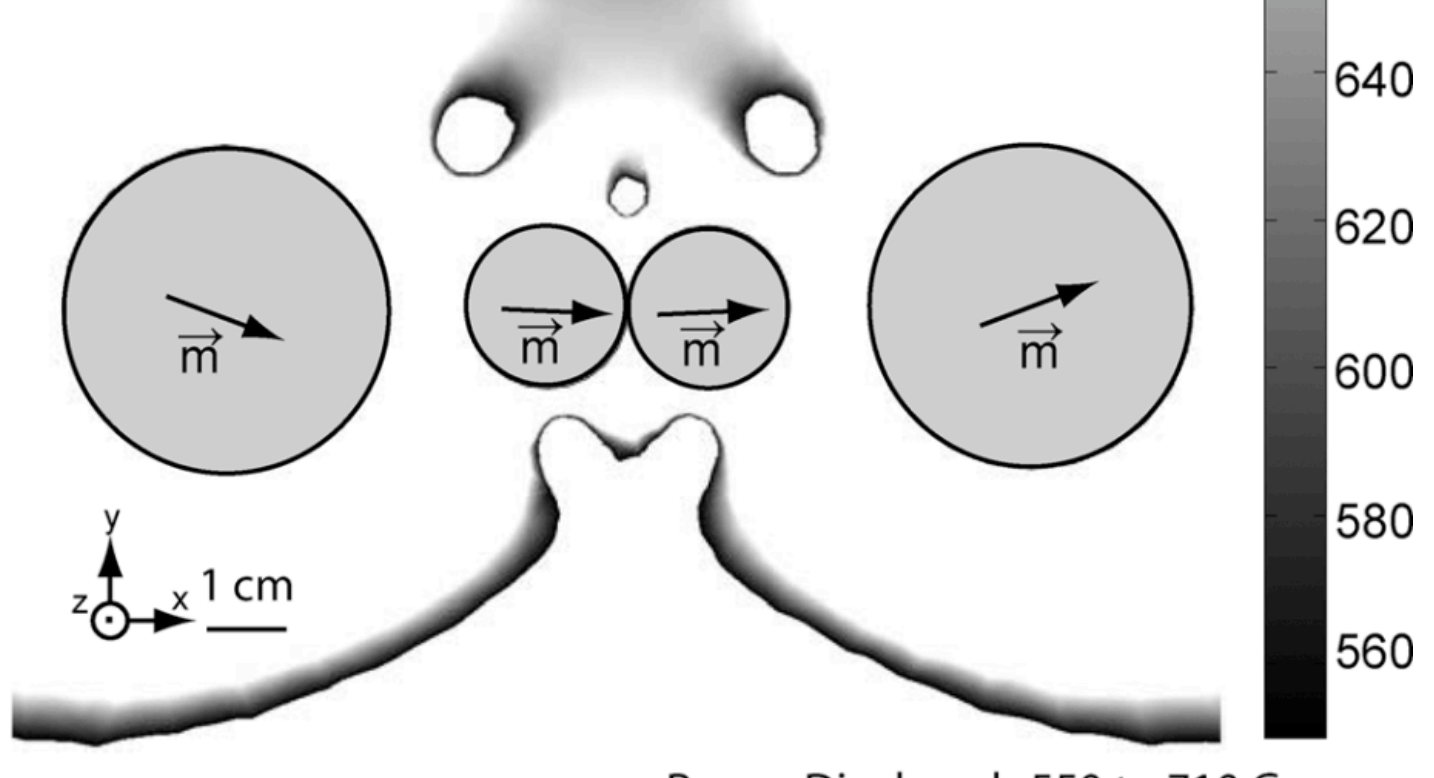

Range Displayed: 550 to 710 Gauss 
Fig. 4. Optimal magnet rod orientations for our optimal magnet design for a range of sensitive volume distances $d_{t}$, and for finite $(18 \mathrm{~cm})$ and infinite length rod implementations with uniform remanent fields of $1.3 \mathrm{~T}$. (i) Homogeneous regions plotted over the slice bisecting the magnet rod lengths produced by finite length rods. The shaded regions indicate where the field's magnitude varies within a part per ten and a part per hundred of the optimal target field strength. The array produces slightly larger homogeneous regions for sensitive volumes near the original target region $\left(d_{t}=3.5 \mathrm{~cm}\right)$. (ii) Field strength of the sensitive volumes. The finite length rods produce sensitive volumes with field strengths that are a fraction of their infinite length rod equivalents which decreases with distance $\left(50 \%\right.$ at $\mathrm{d}_{\mathrm{t}}=$ $3.0 \mathrm{~cm}$ vs $40 \%$ at $\mathrm{d}_{\mathrm{t}}=10.5 \mathrm{~cm}$ ). (iii) Inhomogeneity quantified as the average magnitude of the difference between the actual and target field over the target region normalized to the optimal target field strength. Sensitive volumes produced by the finite rods are slightly more inhomogeneous than for the infinite length rods. The algorithm yields inhomogeneous solutions for $\mathrm{d}_{\mathrm{t}}<2.9 \mathrm{~cm}$ for these target regions. (iv) Optimal rod orientations. The rod angles are illustrated within the Magnet Arrangement portion of $i$. The optimal angles for the finite and infinite length rods match for homogeneous systems with $\mathrm{d}_{\mathrm{t}}<4.5 \mathrm{~cm}$, indicating a region where our finite rod implementation of 2D dipoles is effective.
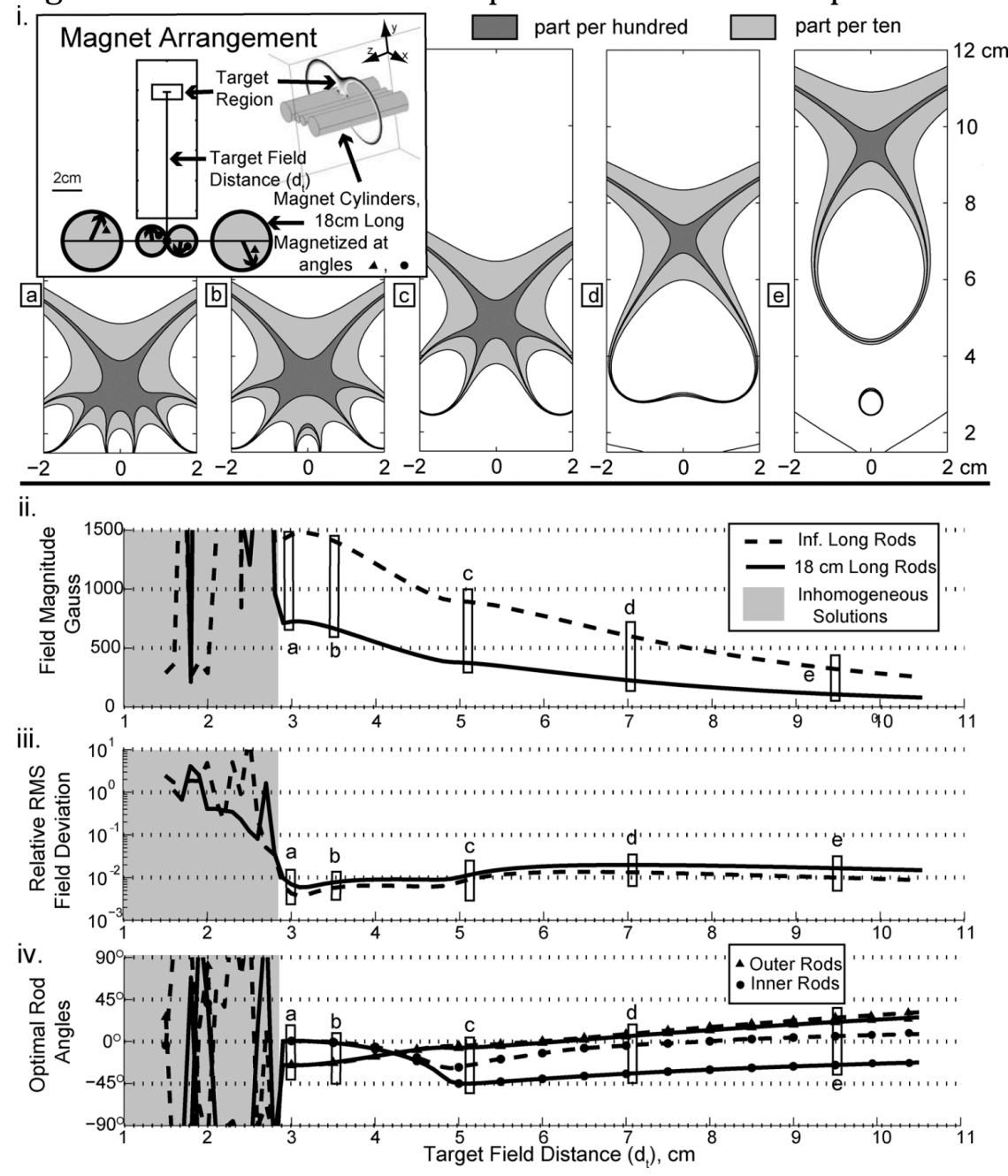
Algorithm 1 Determining the appropriate weighting for the "field-zeroing" region

1: Choose a representative set of dipole positions $\left\{x_{i}\right\}_{0}$.

2: Calculate the target field matrix and vector, $\mathbf{M}$ and $\mathrm{m}$, and the field zeroing matrix $\mathbf{M}_{\mathbf{z}}\left(\mathrm{m}_{\mathbf{z}}=0\right)$ from (5).

\section{3: repeat}

4: Appropriately set or adjust the scaling factor for the field zeroing region, $f_{z}$.

5: Calculate the effective cost function to be minimized.

$$
\mathbf{M}_{\text {eff }}=\left(1-f_{z}\right) \mathbf{M}+f_{z} \mathbf{M}_{\mathbf{z}}, \mathrm{m}_{\mathrm{eff}}=\left(1-f_{z}\right) \mathrm{m} \text {. }
$$

6: Determine the optimal coefficients, $g_{\text {opt }}=\mathbf{M}_{\text {eff }}^{-1} \mathrm{~m}_{\text {eff }}$.

7: until The homogeneity falls within the desired range. 


\section{Algorithm 2 Global Search: Optimal Positions} of the Dipole Array

1: Determine the appropriate zero-field weighting, $f_{z}$ as in Algorithm 1.

2: Determine the distinct sets of dipole positions, $\left\{x_{k}\right\}_{l}$, to be tested.

3: for all $\left\{x_{k}\right\}_{l}$ do

4: Calculate the target field matrix and vector ( $\mathbf{M}$ and $\mathbf{m})$ and the zero-field matrix $\left(M_{z}\right)$ where $m_{z}=0$ from (5).

5: Calculate the effective cost function to be minimized.

$$
\mathbf{M}_{\text {eff }}=\left(1-f_{z}\right) \mathbf{M}+f_{z} \mathbf{M}_{\mathbf{z}}, \mathbf{m}_{\text {eff }}=\left(1-f_{z}\right) \mathrm{m} \text {. }
$$

6: Determine the optimal coefficients, $\mathrm{g}_{\text {opt }}=\mathrm{M}_{\text {eff }}^{-1} \mathrm{~m}_{\text {eff }}$.

7: Calculate the approximate homogeneity, $C$, the implementation's field strength, and its resilience to variations, $\left|\nabla_{\mathrm{x}} C\right| \approx \sqrt{\sum_{\alpha}\left(\left[C\left(x_{\alpha}+\delta\right)-C\left(x_{\alpha}\right)\right] / \delta\right)^{2}}$ and $\left|\nabla_{\mathrm{g}} C\right| \approx \sqrt{\sum_{\alpha}\left(\left[C\left(g_{\alpha}+\delta\right)-C\left(g_{\alpha}\right)\right] / \delta\right)^{2}}$ where $C$ is the cost function in (4) for the main target field.

\section{8: end for}

9: Rank the systems for optimal homogeneity, efficiency and stability.

10: Choose the best compromise system. 
Algorithm 3 Optimizing With Multiple Quadratic Constraints

1: Generate the constraint matrices and scalars, $\mathbf{K}_{j}$ and $\alpha_{k}$.

2: Calculate the target field matrix and vector, $\mathbf{M}$ and $\mathrm{m}$, from (5).

3: while the constraints (8) are not obeyed. do

4: Calculate the first order Lagrange multiplier solution matrices and vectors: L, $A$ and $p$ from (13).

5: Find the first order approximations to the Lagrange multiplier solution vectors $\lambda_{p}$ and $\lambda_{\mathrm{A}}$ from (16).

6: Determine the relative field-strength $s$ which allows for the minimal applied constraint from (17).

7: if $\lambda_{\alpha} \cdot \lambda_{p} \leq 0$ then

8: Quit. Inform the user of the condition and return the previous iteration's solution for $\lambda$ and gopt

9: end if

10: Calculate the Lagrange multiplier, $\lambda$, as in (16).

11: Add in the Lagrange multipliers into the solution matrix, M. See (14). \{This new matrix now replaces the previous one for future loop iterations.

12: Calculate the optimal coefficients. $\mathrm{g}_{\mathrm{opt}}=\mathbf{M}^{-1}(\mathrm{sm})$.

\section{3: end while}

